\title{
MEDICAL AND SURGICAL APPLICATIONS OF FELS
}

\author{
Benedikt Jean, MD, PhD (Invited Paper) \\ Experimental Ophthalmic Surgery, University of Tübingen, FRG
}

Abstract: Every medical laser application is based upon the compromise between efficiency and collateral adverse effects.The optimizing strategies of preclinical investigations of IR lasers can be dramatically improved by using FEL technology, allowing the free combination of wavelength, pulselength and energy. The new approach is illustrated by the results from photoablation of three different types of tissue (cornea, dental hard substances and atheromatous plaques). FEL technology enables the optimizing of physical and biological parameters and reducing risks while minimizing time and costs involved.

\section{INTRODUCTION}

Among the many laser/tissue interactions which have been introduced into microsurgery over the past 15 years, photoablation, the contact free removal of tissue is probably the most spectacular: Never before have we been able to remove tissue with a precision better than one micron, far beyond the resolution of the surgeon's naked eye. In ophthalmic microsurgery, techniques to reshape the cornea, to correct myopia, to remove pathologic tissue on a micron, - or submicron scale have been developed with the $193 \mathrm{~nm}$ excimer laser [1].

Photoablation is possible at wavelengths ranging from the far-UV to the Far-IR. The typical fluences required, range from $180 \mathrm{~mJ} / \mathrm{cm}^{2}$ for the far UV to $1 \mathrm{~J} / \mathrm{cm}^{2}$ in the $\mathrm{mid}$ IR. Photoablation has been achieved with pulselengths ranging from femtoseconds to microseconds and, apart from the three decisive parameters (pulselength, wavelength and fluence) the pulse repetition rate influences the roughness of the ablated surface. The basic principles of photoablation however, have not yet been investigated systematically over a wider wavelength range [2].

In the past, the preclinical investigation of new laser sources depended upon their technical availability: pulselength, wavelength and energy are technically related to each other and they allowed no or only minimal variation of the parameters. Laser surgical procedures for fistulation surgery in ophthalmology, angioplasty, dental surgery, a.s.o. were iteratively optimized, as a function of the mere availability of laser sources. The Deutsche Forschungsgemeinschaft ( German Research Council) alone, has invested a total of 21 million marks (15 million $\$$ ) in experimental laser devices for medicine over a 7-year period (1985-92) for investigational laser equipment (data provided by DFG).

Any medical laser application is based upon the compromise of efficiency and the correlated adverse effects of each set of laser parameters. The surgical requirements, however, are often contradictory: A high ablation rate is required in dental surgery, while it must be minimal to reshape the human cornea; cutting through vascularized tissue (e.g. brain surgery) requires some amount of surface coagulation (thermal "damage") in order to achieve hemostasis while for avasular tissues, wound healing is best when there is no thermal damage. Higher pulse repetition rates reduce surface roughness while, at the same time they increase the overall thermal load on the target site. The absorption coefficient of the target material not only determines the penetration depth of the radiation but also the ablation related shockwave.

Since the FEL has been made available for biomedical research [3], its unique possibility of independently combining wavelength tuneability with any energy and any pulselength allows a new approach. In this manner it is the ultimate research tool for preclinical investigations of new laser sources or new combinations of parameters.

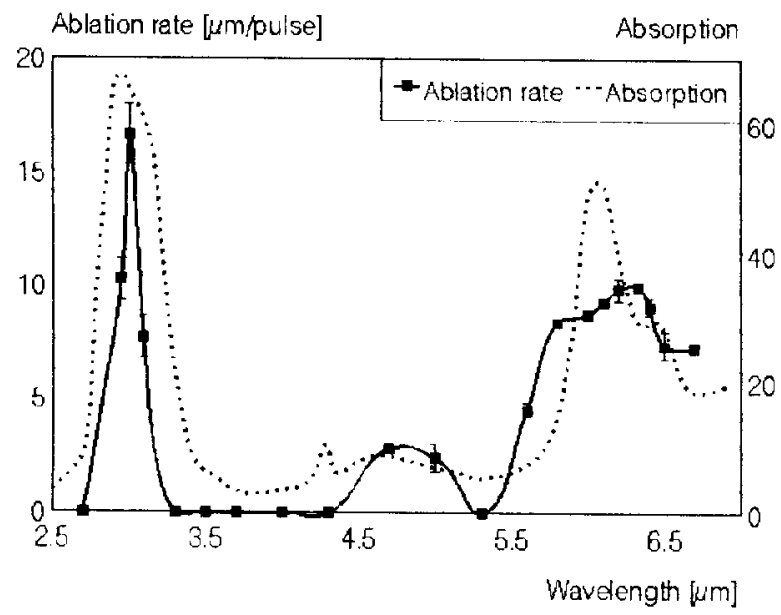

Fig.1: Ablation rate as a function of wavelength

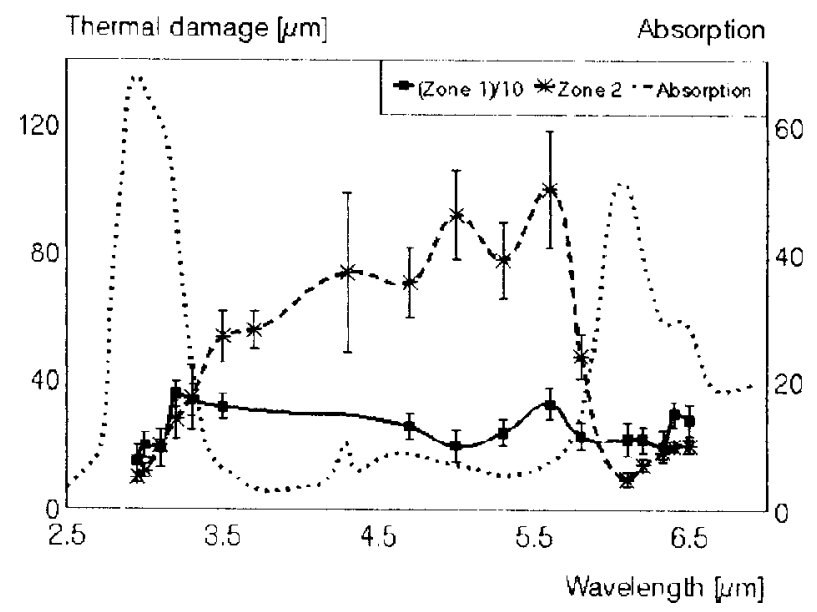

Fig. 2: Thermal damage as a fucntion of wavelength 


\section{RESULTS}

As a typical example for <soft> tissue, with high water content, corneal tissue was chosen to perform the first systematic investigations of IR photoablation with the FEL at Vanderbilt University, between $2.7 \mu \mathrm{m}$ and $6.5 \mu \mathrm{m}$ thus including the two water absorption bands around $3 \mu \mathrm{m}$ and $6 \mu \mathrm{m}$.

The ablation efficiency (Fig 1), as measured by the depth of the ablation crater, is found to be related to the absorption spectrum of the material [4]: at high absorption, ablation efficiency is high, at minimal absorption it is lowest for aconstant fluence. However, even at minimal absorption, ablation is possible, but with the disadvantage of extrem thermal damage.

The collateral thermal damage is relatively uniform in the IR but markedly more pronounced than in the UV. Collateral thermal damage, measured by the extent of the eosinophilic zone (Fig. 2, zone 2) at the bottom of the ablation area, is inversely related to target material's absorption [5].

The water absorption spectrum beyond $6 \mu \mathrm{m}$ shows an increase up to $15 \mu \mathrm{m}$ and decreases slightly between 15 and $50 \mu \mathrm{m}$. This spectral range is covered by the FELIX Free-Electron Laser at FOM, Nieuwegein/the Netherlands. According to our expectations, the first sucessful photoablation in the far IR was performed in corneal tissue at $15 \mu \mathrm{m}$ and $70 \mu \mathrm{m}[6]$.

The relevance of those findings lie in the fact that, for the first time, photoablation was investigated over a wide range of wavelength variation, providing a solid basis for the development of new and the verification of existing models, adding a predictive element to shorten the preclinical optimizing strategy for new laser sources. As a next step, variation of pulselength will be studied as soon as FEL technology permits.

It has recently been proved that the FEL can also be used as an ideal stress wave generator, allowing the study of shock wave related damage in vitro [8]; this approach may provide the first systematic access to the assessment of pressure related cellular damage and its relevance in surgery.

Apart from the predominant water absorption in the human cornea (with its $85 \%$ of water), at $6.45 \mu \mathrm{m}$ the amid II collagen absorption peak allows selective interaction with the incident laser light. This suggestion was the first to be made with regard to selective corneal photoablation in the IR [9].

Dentin and enamel are typical target materials for the study of photoablation with the FEL in bone-like biological materials with low water content: Presently the $2.94 \mu \mathrm{m}$ Er:YAG laser uses the $30 \%$ water content of enamel and dentin as a main absorber in order to achieve photoablation.
The highest absorptions, however, are around $9.5 \mu \mathrm{m}$ for hydroxyapatite, the main constituent (approx. 50\%) of enamel and dentin. A major problem of the $2.94 \mu \mathrm{m}$ radiation is the appearance of thermally induced cracks in the enamel around the ablation site, leading to caries, since they provide favourable environment for bacteria. The $9.5 \mu \mathrm{m}$ wavelength was investigated with FELIX: As expected, the ablation efficiency was maximal and the collateral thermal damage minimal at maximal absorption (Fig.3). At maximal absorption, the penetration depth is minimal and thus the volume of tissue heated per puls is minimal too [14]. As a consequence, the appearance of thermal cracks was also minimal, their size being beyond the diameter of bacteria. Furthermore, at $9.5 \mu \mathrm{m}$, the ablation threshold requires a minimal fluence; this, in return, is an element of selectivity limiting photoablation to enamel/dentin while neighbouring gingiva cannot be ablated. This adds an element of inherent safety to the surgical use of this wavelength.

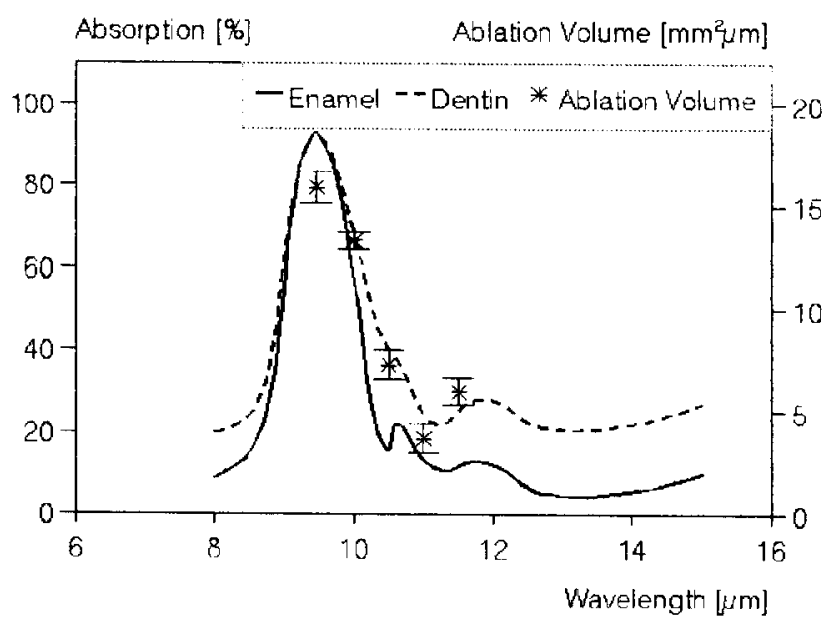

Fig. 3: Absorption spectrum of dentin and enamel and ablated volume

Removal of atheromatous plaques for recanalization of obliterated cardiac vessels (laser angioplasty) is another minimally invasive surgical task with highest socioeconomic relevance. So far only a few selected wavelengths, available with solid state lasers, have been tried in the IR. While the ablation efficiency should be high, the stress wave induced damage to the vessel wall should be minimal and perforation of the vessel, a deadly complication, must be avoided under all circumstances. As plaques show a rather inhomogenous composition (apatite and cholesterol), the "selectivity" of the absorption for the materials to be removed must be high enough to contrast with the absorption properties of the underlying vessel wall (high watercontent) to avoid perforation. Fig. 4 show that the efficiency as well as the selectivity criterion (Fig.5), can be met by using $9.5 \mu \mathrm{m}[15]$. 


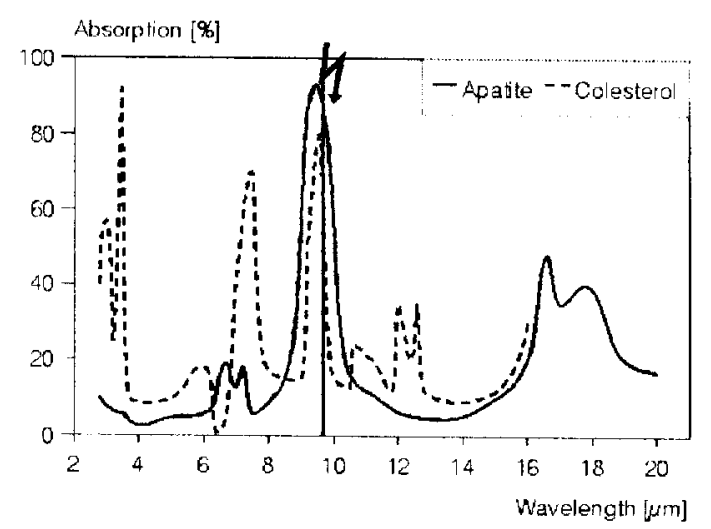

Fig. 4: Absorption spectrum of colesterol and apatite

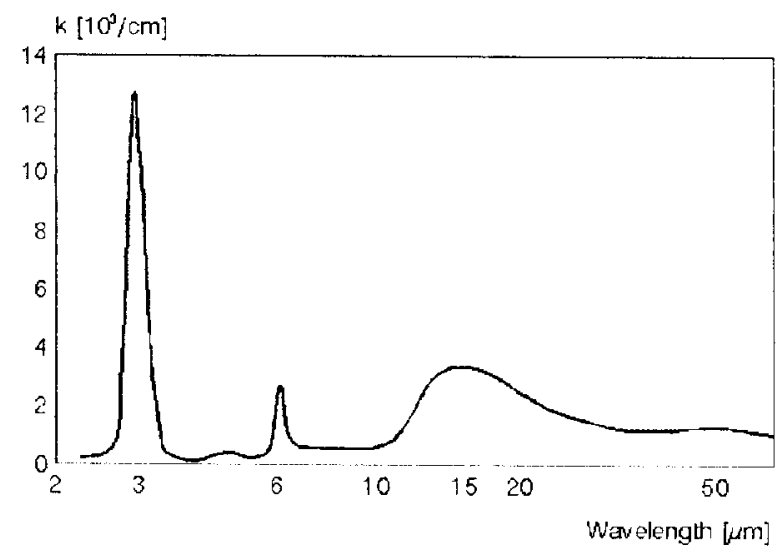

Fig. 5: Absorption coefficient of (ocean) water

As the pressure gradient over the time of the shock wave is related to the target material's absorption, the incident radiation can be tuned off the absorption peak to reduce the harmful collateral pressure gradient which causes micro lesions on the vessel endothelia eventually triggering thrombosis and restenosis. Such studies are in progress.

Discrimination of different tissues which cannnot be targeted selectively by specific absorbers (e.g. if they all have a high water content) is another central goal of microsurgery: a non contact photoacustric spectroscopy (NCPAS) set up, recently suggested for <smart> laser controle [10] captures the sound wave emitted, rather than the pressure signal from the ablation site. It has proved its feasibility to assess the ablation rate, the ablation zone diameter, the fluence and, in particular, its ability to disciminate between different target materials by on-line measurement of the center gravity of the FT signal. In this manner NCPAS measures the absorption coefficient of every ablated volume of tissue; it can be used to stop a laser automatically when it hits a tissue, not to be removed (e.g. a nerve in connective tissue). The FEL was used to study the photoacustic signal as a function of absorption coefficient in the IR [11].
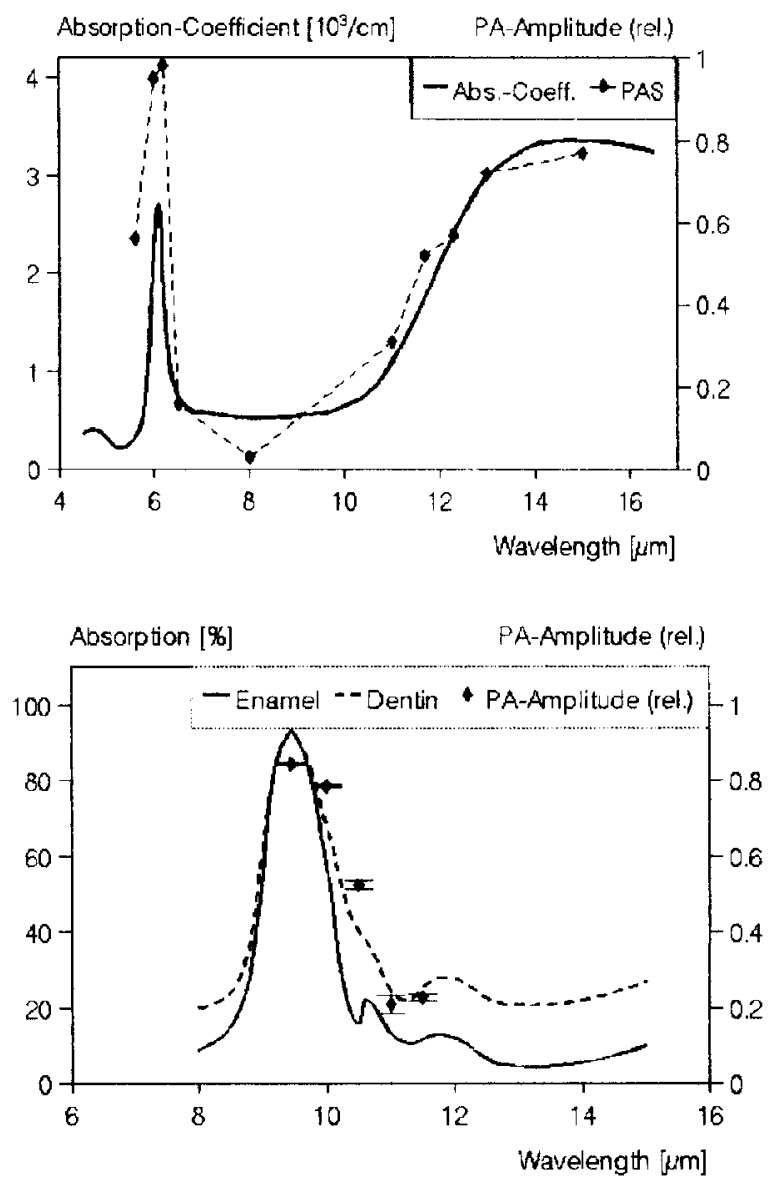

Fig.6: Amplitude of photoacustic signal as a function of wavelength (target material: cornea (top); dentin (bottom)).

Never before have we been able to study efficiency and adverse effects or any of the compromises of a new set of laser parameters so carefully in vitro. As another consequence, we can now minimize surgical risks for the first in-vivo applications on patients dramatically; at the same time, the FEL enables us to minimize costs and time invested in experimental and preclinical applications in medicine and surgery.

The biological adverse effects, rather than the physical parameters describing efficiency and related damage, are the decisive criteria in the assessment of the usefulness of new combinations of parameters for specific medical and surgical applications. The study of such biological adverse effects and the cell's sensitivity to them, however, requires a large data basis, in order to integrate the wide variation of biological parameters. While radiation biology has been systematically investigating the effects of ionizing radiation for years, very little is known about the interference of coherent laser light with living cells.

Advanced automated flow-cytometry [12] allows the assessment of such effects on a large number of cells in very short time by analysing intracellular fluorescent markers, tracing and measuring simultaneously 6 key metabolites. A self classifying software, developed by 
VALET [13] identifies typical damage patterns and correlates them to biochemical pathways as a function of the external stimuli, e.g. the incident laser radiation. A recent pilot study, (supported by the US-ONR), performed in the Far UV and mid IR proved the feasibility of the approach, to assess distinct biochemical damage patterns (Jean, Valet in preparation).

\section{CONCLUSION}

The FEL as a research tool in biomedical laser research allows a new approach to the optimization of laser/tissue interactions by applying a 5 step procedure to bypass conventional time consuming and expensive strategies:

- absorption spectroscopy of the target material

- determination of a "promising" selective interaction with distinct absorbers

- assessment of physical parameters of ablation efficiency and collateral damage

- investigation of related biological and functional biochemical adverse effects.

- preclinical and clinical use

Taken together, FEL technology has opened a new era of systematic and prospective investigation of laser-tissueinteractions and their optimization prior to clinical use. Its enormous potential for reducing time and costs involved and also for minimizing typical risks, associated with its clinical investigations on patients, will play a major role in the development of future minimally invasive surgical techniques in many branches of surgery and interventional medicine.

An important impact on medical laser industries can be expected by reducing typical risks and costs of the conventional iterative optimization strategies which have been the rule so far, thus favouring competitiveness on national and international markets.

Where tuneability of the laser and free combination of parameters for optimal parameter selection are clinically required, the FEL may also gain importance as a therapeutic tool at a few medical and surgical centers. Since 1994, the first medical unit on top of an FEL has been under construction at Vanderbilt University. The FEl's future as a clinical tool, however, will depend upon advances in engineering to reduce costs and complexity of current FEL technology and thus to promote its advantages when competing with alternative technology such a optic parametrical oscillators.

Aknowledgment: We are indebted to Prof. Charles A. Brau, Director of the FEL Center at Vanderbilt University for his continuing support and encouragement and Prof v.d.Wiel and W.v.Amersfoort at FOM-FELIX, Nieuwegein/the Netherlands for granting access to the laser. This work received support from US-ONR grant \# 00014-91-0109 and the European Union HCM grant ERB 4050 PL940877.

\section{REFERENCES}

[1] S. L. Trokel, R. Srinivasan, B. Braren. Excimer laser surgery of the cornea. Am J Ophthalmol, vol. 96, pp. 710 (1983).

[2] T. Bende, B. Jean, M. Matallana, T. Seiler, Ch. A. Brau. Photoablation with the Free-Electron Laser between 2.8 and 6.7 microns wavelength. Invest Ophthalmol (suppl), 34, 4, pp. 1246 (1993).

[3] C. A. Brau. The Vanderbilt University Free Electron Laser Center. Nuclear Instruments and Methods in Physics Research, A 3183R-1 N.Y.Elsevier Science Publisher (1992).

[4] B. Jean ,T. Bende. Photoablation with the FreeElectron Laser between 2.7 and $6.7 \mu \mathrm{m}$. Corn Refr surg, vol.10, 4, pp. 433-438 (1994).

[5] T. Bende, R. Walker, B. Jean. Photoablation in Porcine Corneas with the Free-Electron Laser between 2.7 and $6.7 \mu \mathrm{m}$ wavelength - Thermal Collateral Damage. Corn Refr Surg, vol. 11, 2, pp. 129-136 (1995).

[6] B. Jean, R. Walker, T. Oltrup, T. Bende, W.v. Amersfoort. Free Electron Laser (FEL): Photoablation, Thermal Gradients and Damage in the FAR IR $(10 \mu \mathrm{m}$ to $65 \mu \mathrm{m}$ ). Invest Ophthalmol (suppl), 35, 4, pp. 2155 (1994).

[7] M. Ostertag, R. Walker, T. Bende, B. Jean. Optimizing Photoablation Parameters in the Mid IR - a Predictive Model for the Description of Experimental Data. SPIE's International Symposium of Biomedical Optics '95 (supp) accepted (1995).

[8] D. C. Lamb, L. Reinisch, J. Tribble, R. H. Orsoff ,T. J. Flotte, A.G. Doukas. The FEL As The Ideal Stress-Wave Generator. SPIE's International Symposium of Biomedical Optics '95 (supp) (1995).

[9] G. Edwards, L. Regan, M. Copeland, L. Reinisch, J. Davidson, B. Johnson et al. Tissue ablation by a FreeElectron Laser tuned to the amide II band, Nature, vol. 371, pp. 416-419 (1994).

[10] B. Jean ,T. Bende, M. Matallana. Non Contact Photoacustic Spectroscopy. German J Ophthalmol, vol 2, pp. 404-408 (1993).

[11] B. Jean, M. Matallana, M. Ostertag, R. Walker,T. Bende, W.v. Amersfoort IR Laser photoablation of Cornea Measured by Noncontact Photoacustic Spectroscopy. Invest Ophthalmol (suppl), 36, 4, pp. 987, 4562-543 (1995).

[12] G. Valet, H. H. Warnecke, H. Kahle. Automated Diagnosis of Malignant and Other Abnormal Cells by Flow Cyrtometry using DIAGNOSI program systems. In: Clinical Cytometry and Histometry; G. Burger, JS. Ploem, K. Goerttler (eds). Academic Press London, pp. 58-65 (1987). 
[13] G. Valet, M.Valet, D. Tschöpe, H. Gabriel, G. Rothe, W. Kellermann, H. Kahle. White Cell and Thrombocyte Disorders. Clin Flow Cytom. vol. 677, Ann. NY Acad of Sci (1993).

[14] M. Ostertag, R. Walker, H. Weber, W.v. Amersfoort, B. Jean. Photoablation of Dental Hard Substances at the Absorption Maximum of $9.4 \mu \mathrm{m}$ of Hydroxyapatite. International Free-Electron Laser Conference and 1st Annual FEL User Workshop, August, Stanford (1994).

[15] M. Ostertag, R. Walker, K. Karsch, M. Wehrmann, B. Jean. Photoablation on Atherosclerotic Plaque with 9.5 $\mu \mathrm{m}$ Wavelength. International Free-Electron Laser Conference and 2nd Annual FEL User Workshop, August, New York (1995); accepted. 